Old Dominion University

ODU Digital Commons

Physics Faculty Publications

Physics

$11-2002$

\title{
Simultaneous Trapping of Rubidium and Metastable Argon in a Magneto-Optical Trap
}

\author{
C. I. Sukenik \\ Old Dominion University, csukenik@odu.edu
}

H. C. Busch

Old Dominion University

Follow this and additional works at: https://digitalcommons.odu.edu/physics_fac_pubs

Part of the Atomic, Molecular and Optical Physics Commons

\section{Repository Citation}

Sukenik, C. I. and Busch, H. C., "Simultaneous Trapping of Rubidium and Metastable Argon in a Magneto-Optical Trap" (2002). Physics Faculty Publications. 12.

https://digitalcommons.odu.edu/physics_fac_pubs/12

\section{Original Publication Citation}

Sukenik, C.I., \& Busch, H.C. (2002). Simultaneous trapping of rubidium and metastable argon in a magneto-optical trap. Physical Review A, 66(5), 51402. doi: 10.1103/PhysRevA.66.051402

This Article is brought to you for free and open access by the Physics at ODU Digital Commons. It has been accepted for inclusion in Physics Faculty Publications by an authorized administrator of ODU Digital Commons. For more information, please contact digitalcommons@odu.edu. 


\title{
Simultaneous trapping of rubidium and metastable argon in a magneto-optical trap
}

\author{
C. I. Sukenik and H. C. Busch \\ Department of Physics, Old Dominion University, Norfolk, Virginia 23529
}

(Received 15 August 2002; published 15 November 2002)

\begin{abstract}
We have simultaneously confined rubidium and metastable argon in a dual-species magneto-optical trap (MOT). Here a binary mixture of atomic species from different groups of the periodic table have been optically confined at ultracold temperatures. We describe the apparatus and characterize the individual, single species MOTs and the dual-species MOT. Both fluorescence and ion production are monitored. With both species trapped, we observe $\sim 5 \times 10^{6}{ }^{85} \mathrm{Rb}$ atoms and $\sim 2 \times 10^{6}{ }^{40} \mathrm{Ar} *$ atoms. Realization of the dual-species trap opens the way for detailed studies of Penning and associative ionization, photoassociative spectroscopy, and eventually for the production of bound, ultracold RbAr molecules.
\end{abstract}

DOI: 10.1103/PhysRevA.66.051402

PACS number(s): 32.80.Pj, 33.80.Ps

Recent advances in ultracold molecule production are opening new frontiers of research in low-temperature physics and chemistry. Unlike atoms, molecules do not possess an optical cycling transition and quickly become decoupled from a light field if the standard cooling and trapping techniques used for atoms are applied. As a result, other methods are needed to produce ultracold molecules. To date, a number of techniques to produce cold molecules have been demonstrated including electrodynamic deceleration of polar molecules [1], sympathetic cooling with a helium buffer gas [2], and photoassociation of ultracold atoms in a magneto-optical trap (MOT) [3] or Bose-Einstein condensate [4]. Photoassociation uses well-established methods to cool the translational energy of atoms which are then brought together to form a molecule. Initial experiments demonstrated the feasibility of photoassociating atoms into molecules producing homonuclear dimers, the simplest pair to produce. Recently, attention has turned to the production of heteronuclear dimers for low-temperature physics studies [5] as possible candidates for quantum computation schemes [6], and for parity violation measurements [7]. Because atoms are photoassociated during collisions, the details of the molecule formation process depend strongly on the ultracold collision dynamics which are rather different for the two classes of dimers. This is because for low partial-wave scattering, the first excited state of homonuclear dimers with internuclear separation $R$ is characterized by a long-range $1 / R^{3}$ resonant dipole interaction, whereas in the heteronuclear case it is the much shorter range $1 / R^{6}$ van der Waals interaction which typically dominates.

In this paper we report on the realization of a dual-species MOT which simultaneously confines alkali-metal $\left({ }^{85} \mathrm{Rb}\right)$ and metastable noble-gas ( $\left.{ }^{40} \mathrm{Ar}^{*}\right)$ atoms. Little is known about the interactions of such a mixture at low temperature. In fact, to our knowledge this is the first time that two species from different groups of the periodic table have been confined simultaneously in a MOT. We have constructed the dual MOT with the eventual goal of producing ultracold ground state $\mathrm{RbAr}$, a weakly bound van der Waals molecule. We observe $\sim 5 \times 10^{6}{ }^{85} \mathrm{Rb}$ atoms and $\sim 2 \times 10^{6}{ }^{40} \mathrm{Ar}^{*}$ atoms in a cloud with an approximate diameter of $500 \mu \mathrm{m}$. We have also observed a modest interaction between the $\mathrm{Rb}$ and $\mathrm{Ar} *$ atoms confined in the dual MOT. The coexistence of the two species under nominal MOT conditions was not a foregone conclusion given that no information is available on crossspecies ionization rates at ultracold temperatures. Hence the observation of a robust dual-species MOT containing $\mathrm{Rb}$ and Ar* has important positive implications for further studies of photoassociation and molecule formation.

In addition to the promise of producing a weakly bound heteronuclear molecule, the $\mathrm{Rb}+\mathrm{Ar}^{*}$ system contains some features that differentiate it from single-species traps. As noted above, the homonuclear and heteronuclear collision dynamics are considerably different at ultracold temperatures, where the details of the long-range part of the potential are very important. In addition, with the considerable internal energy of the Ar*, interspecies ionizing collisions can be studied in detail and excited-state reaction products can be produced. Even-numbered isotopes of the noble gases do not have hyperfine structure, thereby allowing for the detailed study of the role of hyperfine structure of just one of the collision partners. And finally, for suitable $s$-wave scattering lengths, it may be possible to employ sympathetic cooling of the noble gas by alkali metal atoms as an alternative route to achieving Bose-Einstein condensation of noble gases or to produce binary Bose-Einstein condensates.

The dual MOT is produced in a stainless steel, differentially pumped, vacuum chamber with three regions. $\mathrm{Ru}-$ bidium atoms are loaded into the MOT from a background vapor which is produced by running a current $(\sim 3.4 \mathrm{~A})$ through an alkali-metal dispenser (SAES Getters). The metastable argon is loaded from an atomic beam. The MOT region is pumped by a 50-1/s turbo pump and an $11-1 / \mathrm{s}$ ion pump. The pressure in the MOT chamber is monitored by the ion pump current. A 170-1/s turbo pump is used in the source region, and a 50-1/s turbo pump is used in conjunction with a liquid-nitrogen cold trap in an intermediate region, between the MOT and the source. Anti-Helmholtz coils, placed external to the chamber, provide an axial magnetic-field gradient of $10 \mathrm{G} / \mathrm{cm}$ at the center of the trapping region.

Metastable atoms are produced in a rf discharge, which operates at a frequnecy of $155 \mathrm{MHz}$ and closely follows the design of Chen et al. [8]. We estimate the metastable flux to be near the $4 \times 10^{14} \mathrm{~s}^{-1} \mathrm{sr}^{-1}$ reported in Ref. [8]. The discharge frequency is very close to the 2-m amateur radio band, allowing us to use an inexpensive rf amplifier (Mirage 


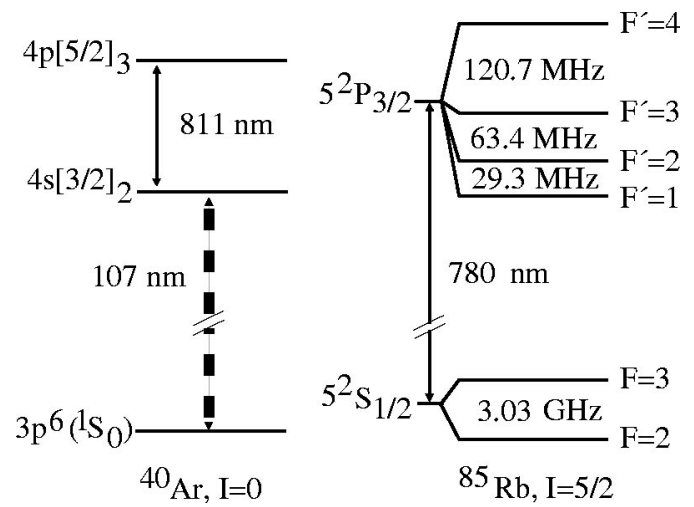

FIG. 1. Partial energy-level diagrams for ${ }^{85} \mathrm{Rb}$ and ${ }^{40} \mathrm{Ar}$. The dotted line indicates an electric dipole forbidden transition that gives rise to the metastability of the $4 s[3 / 2]_{2}$ state.

B-34-G) and antenna tuner (MFJ-921) to operate the discharge. Ions are removed from the atomic beam by a pair of electric-field plates. The atomic beam (containing Ar and $\left.\mathrm{Ar}^{*}\right)$ then enters a Zeeman slower, where the Ar* atoms are translationally cooled in one dimension before entering the MOT capture zone. The Zeeman slower is constructed using a single layer of 14 gauge magnet wire that is wound, with variable pitch, around a standard 1.5 inch outer diameter stainless steel tube. A water cooled brass jacket is placed around the Zeeman coil.

Separate laser systems are used for each species. An energy-level diagram for both atoms is shown in Fig. 1. A Sanyo DL7140-201 laser diode is injection locked to an external-cavity diode laser master (also a Sanyo DL7140201) to produce the trapping and repumping light for $\mathrm{Rb}$ at $780 \mathrm{~nm}$. The repumping frequency is obtained by direct modulation of the slave current through a bias- $T$ junction to produce sidebands at the required frequency [9]. Typically, the sideband intensity is $10 \%$ of the fundamental. A Coherent 899-01 Ti:sapphire laser with a Coherent 895 etalon assembly (to narrow the linewidth) is used to produce the $811-\mathrm{nm}$ trapping light for ${ }^{40} \mathrm{Ar}^{*}$. Note that with no hyperfine structure in ${ }^{40} \mathrm{Ar}$, a repumper laser is not required.

We have added a piezoelectric driven mirror (tweeter) to the Ti:sapphire laser to allow for frequency stabilization. Without a reference cavity or some of the more sophisticated locking techniques often used in narrow-band lasers, we find that the Ti:sapphire laser is susceptible to acoustic noise which translates to pronounced oscillations in the output frequency. The frequency jitter is on the order of the natural linewidth of the trapped atoms, and hence does impact trap performance. We are presently modifying our Ti:sapphire laser to better shield from acoustic perturbations and hence improve the Ti:sapphire locking and Ar* MOT stability.

Both laser are locked using phase-sensitive detection to separate saturated absorption spectrometers. In the case of $\mathrm{Rb}$, a small portion of light from the master laser passes through an acousto-optic modulator (AOM), driven at 80 $\mathrm{MHz}$, and is downshifted in frequency before entering the saturated absorption spectrometer. We then lock the laser to the peak of the $F=3 \rightarrow F^{\prime}=2-4$ crossover, resulting in a trapping frequency red detuned two natural linewidths from the $F=3 \rightarrow F^{\prime}=4$ cooling transition in ${ }^{85} \mathrm{Rb}$. Since there are no crossover peaks in the ${ }^{40} \mathrm{Ar}^{*}$ spectrum, the locking scheme must be slightly different than for $\mathrm{Rb}$. The output of the Ti:sapphire laser is split into three parts. One part passes through an $\mathrm{AOM}$ driven at $80 \mathrm{MHz}$, and is sent to the ${ }^{40} \mathrm{Ar}^{*}$ saturated absorption spectrometer. Unlike the $\mathrm{Rb}$ setup, the light is shifted to the blue of the $4 s[3 / 2]_{2} \rightarrow 4 p[5 / 2]_{3}$ cooling transition in ${ }^{40} \mathrm{Ar} *$. This results in an output frequency of the Ti:sapphire laser which is detuned $80 \mathrm{MHz}$ to the red of the cooling transition. A second AOM then shifts the portion of light used for trapping up by $68 \mathrm{MHz}$ before being sent to the MOT chamber, again resulting in a trapping laser frequency which is two natural linewidths from resonance. A third AOM shifts the final portion of light down by another $80 \mathrm{MHz}$, yielding a total red detuning of $160 \mathrm{MHz}$. This light is then sent to the Zeeman slower.

All light passes through single-mode optical fibers before arriving at the MOT. Because of the proximity of the two wavelengths, identical optics, including wave plates, can be used for the $\mathrm{Rb}$ and $\mathrm{Ar}^{*}$ light. Generally, this is quite beneficial, but it does complicate combining the two beams before sending them into the MOT chamber because dichroic optics for such a small wavelength separation are difficult to fabricate. After exiting their respective fibers, the light for each species is identically collimated and combined on a polarizing beam-splitter cube. This results in a complete spatial overlap with no loss of light, but yields orthogonal polarizations for the two colors. The polarization of both wavelengths is then rotated by $45^{\circ}$ with a half wave plate before impinging on a second polarizing beam-splitter cube which yields two beams each with an equal amount of identically polarized 780-nm and 811-nm light. One beam is sent to the vertical arm of the MOT, and the other beam is further split on a 50-50 nonpolarizing beam-splitter cube and sent to the two horizontal arms of the MOT. Before entering the chamber, the linearly polarized light on each axis is made circular with a $1 / 4$ wave plate. Note that a single wave plate is used for both colors. After exiting the chamber, all three beams are retroreflected after passing through a $1 / 4$ wave plate. Although intensities on the three axes are not the same, the trap operates well. The total laser power available after the output of the fiber is $8 \mathrm{~mW}$ at $780 \mathrm{~nm}$ and $27 \mathrm{~mW}$ at $811 \mathrm{~nm}$. Both beams are expanded to a $1 / e^{2}$ diameter of $1 \mathrm{~cm}$.

The light for the Zeeman slower is also brought to the trapping chamber via a single-mode fiber. After passing through a linear polarizer and 1/4 wave plate, $25 \mathrm{~mW}$ of light propagates along the Ar* atomic beam axis. The $1 / e^{2}$ diameter is $\sim 1 \mathrm{~cm}$ entering the chamber and the beam is slightly focusing as it propagates along the Zeeman slower.

The fluorescence from the trapped $\mathrm{Rb}$ and $\mathrm{Ar}^{*}$ atoms is monitored by separate photomultipliers (Hamamatsu R928), each equipped with a narrow bandpass interference filter (Melles Griot 03 FIL 056 for Rb, and Esco S908100 for $\left.\mathrm{Ar}^{*}\right)$. This allows us to independently observe the behavior of each species. Two charge coupled device (CCD) cameras, placed at right angles to each other, ensure good spatial overlap of the two traps. In addition, a channel electron multipler (Amptek MD-502) is placed $10 \mathrm{~cm}$ from the center of the 


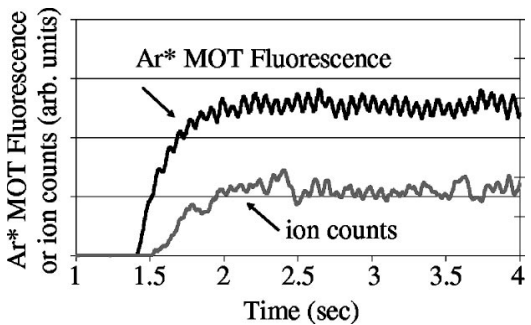

FIG. 2. Ar* MOT loading curve. Both Ar* trap fluorescence and ion production (from ionizing collisions between $\mathrm{Ar}^{*}$ atoms in the MOT) are shown. Oscillations visible in the data arise from laser frequency instability as discussed in the text.

trap to monitor ion production. With the Rb laser blocked we first load the Ar* MOT. A typical loading curve for the Ar* MOT, obtained by measuring the Ar* fluorescence as a function of time, is shown in the upper trace of Fig. 2. Concurrently, we also record the ion counts as shown in the lower trace of Fig. 2. As the MOT loads, both the atom number and the density increase, yielding an increase in Penning and associative ionization resulting from $\mathrm{Ar}^{*}-\mathrm{Ar}^{*}$ collisions [10]. The oscillations visible in the loading curve arise from laser instabilities as discussed above. After the Ar* MOT has loaded, we unblock the $\mathrm{Rb}$ laser, allowing the $\mathrm{Rb}$ trap to form in the presence of the Ar* MOT, as shown in Fig. 3.

An interaction between the ultracold $\mathrm{Rb}$ and $\mathrm{Ar}^{*}$ has been observed. As with single-species traps, light-assisted collisions have been observed to induce trap loss in mixed alkalimetal MOTs. In addition to the dominant trap loss mechanisms observed in alkali systems (such as radiative escape), collisional ionization (Penning and associative) from $\mathrm{Rb}-\mathrm{Ar}^{*}$ collisions is expected to be a major, if not dominant, component of the interspecies trap loss rate. With $11.5 \mathrm{ev}$ of internal energy, a $4 s[3 / 2]_{2} \mathrm{Ar}^{*}$ atom has sufficient energy to ionize a ground-state $\mathrm{Rb}$ atom, whose ionization energy is $4.2 \mathrm{eV}$.

With the experimental conditions reported here, the observed interaction between $\mathrm{Rb}$ and $\mathrm{Ar}^{*}$ is relatively small. We have measured an $8 \%$ decrease in the number of trapped $\mathrm{Rb}$ atoms after the Ar* MOT is allowed to load. Somewhat larger interactions have been observed in dual-alkali metal MOTs containing $\mathrm{Rb}$ and $\mathrm{K}$ [11,12], $\mathrm{Rb}$ and $\mathrm{Na}$ [13], $\mathrm{Rb}$ and Cs [14], and Cs and Li [15]. However, neither our Rb trap

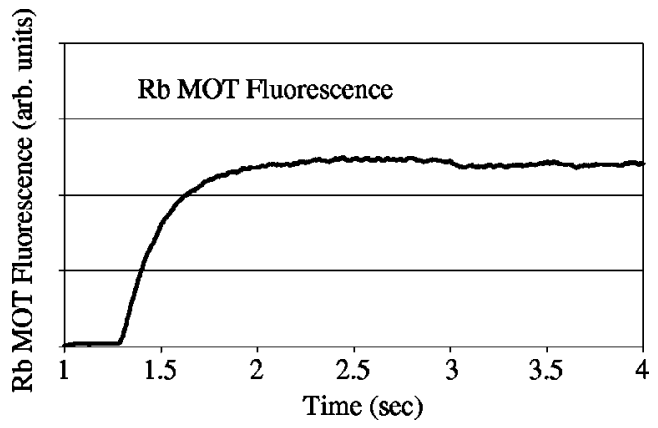

FIG. 3. Fluorescence from the Rb MOT as it loads in the presence of the $\mathrm{Ar}^{*}$ MOT. Fluorescence at $780 \mathrm{~nm}$ is monitored by a photomultiplier tube outfitted with a narrow band interference filter.

nor our $\mathrm{Ar}^{*}$ trap has yet been optimized for density; we expect to observe an increase in the $\mathrm{Rb}-\mathrm{Ar}^{*}$ interaction at different MOT settings. We did not observe a clear decrease in the Ar* MOT signal due to the presence of the Rb MOT, although noise on the Ar* MOT fluorescence (discussed above) prevented us from drawing any conclusions about the reciprocity of trap loss in the dual MOT at this time.

We are presently improving the stability of our Ti:sapphire laser system in order to allow for a detailed study of the interaction of the $\mathrm{Rb}$ and $\mathrm{Ar} *$ MOTs. We wish to fully characterize the collision dynamics of the system and precisely measure the interspecies collisional loss rates. We will also begin, shortly, to search for bound-state structure in the excited states directly above the $\mathrm{Rb} 5 S_{1 / 2}+\mathrm{Ar} * 4 s[3 / 2]_{2}$ level.

In summary, we have realized the first dual-species magneto-optical trap that confines alkali-metal $(\mathrm{Rb})$ and metastable noble-gas ( $\left.\mathrm{Ar}^{*}\right)$ atoms. The two species from different groups of the periodic table have been simultaneously trapped at ultracold temperatures. We observe $\sim 5$ $\times 10^{6}{ }^{85} \mathrm{Rb}$ atoms and $\sim 2 \times 10^{6}{ }^{40} \mathrm{Ar}^{*}$ atoms in a cloud with an approximate diameter of $500 \mu \mathrm{m}$. Realization of the RbAr* dual-species trap opens the way for detailed studies of Penning and associative ionization, photoassociative spectroscopy, and eventually for the production of ultracold $\mathrm{RbAr}$ molecules.

We thank M.D. Havey for useful discussions. This work was supported by Old Dominion University and the Thomas F. Jeffress and Kate Miller Jeffress Memorial Trust.
[1] H.L. Bethlem, G. Berden, A.J.A. van Roij, F.M.H. Crompvoets, and G. Meijer, Phys. Rev. Lett. 84, 5744 (2000).

[2] J.D. Weinstein, R. deCarvalho, T. Guillet, B. Friedrich, and J.M. Doyle, Nature (London) 395, 148 (1998).

[3] N. Vanhaecke, W. de Souza Melo, B.L. Tolra, D. Comparat, and P. Pillet, Phys. Rev. Lett. 89, 063001 (2002); C. Gabbanini, A. Fioretti, A. Lucchesini, S. Gozzini, and M. Mazzoni, ibid. 84, 2814 (2000); A.N. Nikolov, J.R. Ensher, E.E. Eyler, H. Wang, W.C. Stwalley, and P.L. Gould, ibid. 84, 246 (2000); T. Takekoshi, B.M. Patterson, and R.J. Knize, Phys. Rev. A 59, R5 (1999).

[4] C. McKenzie, J. Hecker Denschlag, H. Haffner, A. Browaeys,
L.E.E. de Araujo, F.K. Fatemi, K.M. Jones, J.E. Simsarian, D. Cho, A. Simoni, E. Tiesinga, P.S. Julienne, K. Helmerson, P.D. Lett, S.L. Rolston, and W.D. Phillips, Phys. Rev. Lett. 88, 120403 (2002); R. Wynar, R.S. Freeland, D.J. Han, C. Ryu, and D.J. Heinzen, Science 287, 1016 (2000); E.A. Donley, N.R. Claussen, S.T. Thompson, and C.E. Wieman, Nature (London) 417, 529 (2002).

[5] U. Schloder, C. Silber, and C. Zimmermann, Appl. Phys. B: Lasers Opt. 73, 801 (2001); J.P. Shaffer, W. Chalupczak, and N.P. Bigelow, Phys. Rev. Lett. 82, 1124 (1999).

[6] D. DeMille, Phys. Rev. Lett. 88, 067901 (2002).

[7] M.G. Kozlov and V.V. Yashchuk, Pis'ma Zh. Eksp. Teor. Fiz. 
64, 659 (1996) [JETP Lett. 64, 709 (1996)].

[8] C.Y. Chen, K. Bailey, Y.M. Li, T.P. O’Connor, Z.-T. Lu, X. Du, L. Young, and G. Winkler, Rev. Sci. Instrum. 72, 271 (2001).

[9] R. Kowalski, S. Root, S.D. Gensemer, and P.L. Gould, Rev. Sci. Instrum. 72, 2532 (2001).

[10] See, for example, H. Katori and F. Shimizu, Phys. Rev. Lett. 73, 2555 (1994).

[11] J. Goldwin, S.B. Papp, B. DeMarco, and D.S. Jin, Phys. Rev. A 65, 021402(R) (2002).
[12] L.G. Marcassa, G.D. Telles, S.R. Muniz, and V.S. Bagnato, Phys. Rev. A 63, 013413 (2000).

[13] Y.E. Young, R. Ejnisman, J.P. Shaffer, and N.P. Bigelow, Phys. Rev. A 62, 055403 (2000).

[14] G.D. Telles, W. Garcia, L.G. Marcassa, V.S. Bagnato, D. Ciampini, M. Fazzi, J.H. Muller, D. Wilkowski, and E. Arimondo, Phys. Rev. A 63, 033406 (2001).

[15] U. Schloder, H. Engler, U. Schunemann, R. Grimm, and M. Weidemuller, Eur. Phys. J. D 7, 331 (1999). 